\title{
Anemia and Iron Metabolism in COVID-19
}

\author{
Neha Singh ${ }^{1 *}$, Dinesh Kumar ${ }^{2}$ and Rammilan ${ }^{2}$ \\ ${ }^{1}$ Government Medical College, Budaun, (UP), India \\ ${ }^{2}$ Shobhit Deemed University, Meerut, (UP), India \\ *Corresponding author
}

\section{A B S T R A C T}

\begin{tabular}{|l|}
\hline Ke y w o r d s \\
Anemia, \\
$\begin{array}{l}\text { Hemoglobin, } \\
\text { Ferritin, Iron, } \\
\text { Covid-19 }\end{array}$ \\
\hline Article Info \\
\hline $\begin{array}{l}\text { Accepted: } \\
\text { 20 August } 2020 \\
\text { Available Online: } \\
\text { 10 September } 2020\end{array}$ \\
\hline
\end{tabular}

The coronavirus 2 (SARS-CoV-2) pandemic is viciously spreading through the continents with rapidly increasing mortality rates. Current management of COVID-19 is based on the premise that respiratory failure is the leading cause of mortality. However, mounting evidence links accelerated pathogenesis in gravely ill COVID-19 patients to a hyperinflammatory state involving a cytokine storm. Several components of the heightened inflammatory state were addressed as therapeutic targets Iron metabolism and anemia may play an important role in multiple organ dysfunction syndrome in Coronavirus disease 2019 (COVID-19). We conducted a systematic review and meta-analysis to evaluate biomarkers of anemia and iron metabolism (hemoglobin, ferritin, transferrin, soluble transferrin receptor, hepcidin, hapatoglobin, unsaturated iron-binding capacity, erythropoietin, free erythrocyte protoporphyrine, and erythrocyte indices) in patients diagnosed with COVID-19, and explored their prognostic value. Here we address implications of a possible role for hyper-ferritinemia, and altered iron homeostasis in COVID-19 pathogenesis, and potential therapeutic targets in this regards.

\section{Introduction}

The coronavirus 2019 (COVID-19) pandemic has taken the world by surprise as it viciously spread through the continents with rapidly increasing mortality rates. Current management of COVID-19 is based on the premise that respiratory failure is the leading cause of fatalities (1). Nevertheless, mounting evidence points to drastic systemic events taking place that contribute to accelerated COVID-19 pathogenesis. The "cytokine storm" is a notion that is reportedly hailed as the hallmark of the COVID-19 hyper- inflammatory state (2). Consecutive studies linked COVID-19 related hyper-inflammation to systemic events including hypercoagulability, oxidative stress and altered iron metabolism (3). These events were linked to accelerated pathogenesis in gravely ill COVID-19 patients as highlighted in a recent perspective (1). Several components of the heightened inflammatory state have been proposed as therapeutic targets, particularly IL-6 blockers as drugs of more relevance in COVID-19 management than steroids, however concerns of prolonging viral clearance were stated (4). Ferritin is a 
crucial component of iron metabolism, one of the most ancestral systems of host protection from pathogen infections [3]. Iron is a micronutrient necessary for both energy production at a mitochondrial level and nucleic acid replication at cytoplasmic and nuclear level. For its scarcity in the human body and the fundamental processes in which it is involved, pathogens (bacterial, viral or fungal) compete with the host for iron availability in order to guarantee their own replication. When innate immunity is activated and cytokine cascades start, IL-1 and IL-6 stimulate hepcidin expression in the liver, reducing iron bioavailability by decreasing its gut absorption and hiding it into ferritin, a shell-like molecule deposited in macrophages. These mechanisms have been extensively reviewed in the literature $[5,6,7]$.

With this observational study, we aim to provide a detailed description of iron metabolism and lymphocyte subpopulations in ICU Covid-19 population. It can lead to a better understanding of the underlying physiopathology of this unknown disease, foreseeing some possible alternative therapeutic targets.

\section{Materials and Methods}

\section{Study Population}

This study was approved by the Government of Medical College of Budaun,(UP),India. Oral consent was obtained from the patients, and written informed patient consent was waived by the ethics board of Budaun People's Hospital of Government medical College for quarantine in U.P., India. All patients with COVID-19 enrolled in this research were diagnosed according to the guidance of World Health Organization (WHO) and the National Health Commission of India from February 1 to February 29, 2020 (29).

\section{Diagnostic and Classified Criteria}

Nasopharyngeal swab samples were collected for detection, and reverse transcriptionpolymerase chain reaction was used to detect open reading frame 1aboratory (open reading framelab [ORF1ab]) and nucleocapsid protein (nucleocapsid protein $\mathrm{N}$ ) of SARSCoV-2. Reverse transcription-polymerase chain reaction was carried out as per the protocol issued by the WHO. The SARSCoV-2-positive case confirmation needs to meet the requirement that both the target genes are positive at the same time or that ORFlab is positive in 2 different samples of the same patient. According to the seventh edition of COVID-19's diagnosis and treatment plan issued by the Chinese National Health Commission, patients with COVID-19 are divided into 3 main types according to the following clinical manifestations: (1) mild with fever, respiratory or digestive symptoms, and physician-diagnosed pneumonia by chest computed tomography (CT); (2) severe - one of the following conditions: shortness of breath and respiratory rate $\geq 30$ times/minute, oxygen saturation $\leq 93 \%$ at rest, or chest CT imaging showing lesion progression of more than 50\% within 24-48 hours; (3) critical meet any of the following rules: respiratory failure and need for mechanical ventilation, shock, and any other organ failure needing critical care and treatment (24).

\section{Data source and strategy}

We searched MEDLINE (National Library of Medicine, US), EMBASE (Elsevier, Netherlands), Web of Science (Clarivate Analytics, US), Cochrane (Cochrane Collaboration, UK), the WHO COVID-19 database and Google Scholar (Google, Inc., US) to identify relevant articles. We used search terms related to COVID-19 infection and SARS-CoV-2 virus, and several markers of anemia and iron storage and metabolism, 
including hemoglobin, ferritin, transferrin, soluble transferrin receptor, hepcidin, haptoglobin, unsaturated iron-binding capacity, erythropoietin, free erythrocyte protoporphyrin, red blood cell count (RBC), red cell distribution width (RDW), red cell volume (RCV), mean corpuscular volume (MCV), mean corpuscular hemoglobin $(\mathrm{MCH})$, mean corpuscular hemoglobin concentration (MCHC), reticulocyte count, and reticulocyte index. We also included search terms related to clinical progression of COVID-19, such as prognosis, severity, ICU admission, mortality, risk factors, clinical features, clinical characteristics or predictors. (23)

\section{Study selection and eligibility criteria}

All observational studies (e.g., crosssectional, cohort, and case-control studies), except for case reports and case-series, were included.(22) We included studies that reported on the levels of the biomarkers of iron metabolism, erythropoietin and erythrocyte indices, hemoglobin levels or the prevalence of anemia among COVID-19 patients, or their levels by the clinical outcome of patients with COVID-19. Outcomes of interest include disease severity, admission to intensive care unit, mechanical ventilation, and mortality across all age groups. Studies examining the association between the biomarkers and risk of COVID19 complications (e.g., admission to intensive care unit, death) were also included.(26)

\section{Results and Discussion}

Serum iron does not show significant modifications, but it follows the same trend of TfSat being part of the formula to calculate it. They both remain lower than the normal reference values during the whole infection. It is difficult to hypothesise the reason of their trend without proper laboratory research: the dramatic low levels at ICU admission might be expected, but the following increase during an acute phase of inflammatory response seems counter-intuitive. The described patients arrived in our ICU after a median of 7 to 9 days from symptoms onset. (25) The physiologic progressive restoration of normal TfSat after 1 week of infection and the dysregulated cytokine response might contribute to TfSat elevation. Also, it has been described that acute respiratory distress syndrome (ARDS) causes reduced serum iron levels $[9,10]$. intubated Covid-19 patients suffer of this syndrome, while protective ventilation might theoretically restore normal iron levels. Interestingly, recent studies implicated that ferroptosis, which is the process of programmed cell death mediated by iron-dependent peroxidation mechanisms (11) in inflammatory pathologies, involves multiple organs including liver, kidney, heart and lung (12). Ferroptosis was found to be linked to neurological disturbances including cognitive impairment (13), agueusia and anosmia (taste and smell loss) (14) that are regular manifestations of COVID-19 disease. Iron chelators and ferroptosis inhibitors had protective effects by inhibiting intracellular iron induced lipid peroxidation (15). The impact of iron overload on extra and intracellular mitochondria dysfunction, on microbiota dysbiosis (lungs and gut) (15).

Furthermore, serum coagulability is a major concern in COVID-19 pathogenesis, and rapidly recognized as a key risk factor in susceptible patients $(16,17)$. In the context of the cellular iron overload, it has long been documented that coagulopathy is a hallmark of iron toxicity. Oxidized iron accelerates serum coagulation by interacting with proteins of coagulation cascade (18). Ferritin is a very early and non-specific indicator of inflammation. It resulted to be the first severely elevated biomarker together with lymphopenia [19]: thus, its early dosage in at- 
home symptomatic patients might be extremely useful in individuating those who can benefit of early hospital admission. After its initial rise, ferritin can take longer than a month to normalise after an infection [20]. Thus, it remains elevated during ICU stay of Covid-19 patients. Despite being apparently futile, its dosage constitutes the key element to suspect secondary haemophagocyticlymphohistiocytosis (sHLH). sHLH is a frequently misdiagnosed syndrome related to viral infections and thus of primary importance in this Covid-19 pandemic. Overall, despite unable of a more detailed description, we demonstrate how iron metabolism is deranged in severe forms of Covid-19.(27)

In conclusions we describe iron metabolism, lymphocyte subgroups count and other biomarkers in critically ill Covid-19 patients. This might be relevant for clinicians dealing with critical patients and provide further hints about the pathophysiology of this disease. Iron metabolism has been repeatedly proposed as a potential therapeutic target during infections [21, 22]. Pharmacologic advances have made available safer iron chelators [23]. This testifies the relevance of the topics investigated and how they might contribute to the development of novel therapeutic strategies against Covid-19.

\section{Conflict of interest}

No conflict of interest to declare.

\section{References}

Bessman, N.J., J.R.R. Mathieu, C. Renassia, L. Zhou, T.C. Fung, K.C. Fernandez, et al.

Cassat JE, Skaar EP. Iron in infection and immunity. Cell Host Microbe. 2013; 13:509-19.

Chen T, Wu D, Chen H, Yan W, Yang D, Chen $\mathrm{G}$, et al., Clinical characteristics of 113 deceased patients with coronavirus disease
2019: retrospective study. BMJ. 2020; 368: m1091.

Cortese-Krott, M.M., A. Koning, G.G.C. Kuhnle, P. Nagy, C.L. Bianco, A. Pasch, et al., The reactive species interactome: evolutionary emergence, biological significance, and opportunities for redox metabolomics and personalized medicine Antioxid Redox Signal, 27 (10) (2017), pp. 684-712.

Cossarizza A, De Biasi S, Guaraldi G, Girardis M, Mussini C. Modena Covid-19 Working Group (MoCo19). SARS-CoV-2, the virus that causes COVID-19: cytometry and the new challenge for global health. Cytometry A. 2020; 97: 340-3.

Daher, R., H. Manceau, Z. Karim Iron metabolism and the role of the iron-regulating hormone hepcidin in health and disease Presse Med, 46 (December (12)) (2017), pp. e272-8.

Dendritic cell-derived hepcidin sequesters iron from the microbiota to promote mucosal healing Science (80-), 368 (April (6487)) (2020), pp. 186-189.

Dinc, M.E., A. Dalgic, S. Ulusoy, D. Dizdar, O. Develioglu, M. Topak Does iron deficiency anemia affect olfactory function? ActaOtolaryngol, 136 (July (7)) (2016), pp. 754-757.

Drakesmith H, Prentice A. Viral infection and iron metabolism. Nat Rev Microbiol. 2008;6:541-52.

Fan BE, Chong VCL, Chan SSW, Lim GH, Lim KGE, Tan GB, et al., Hematologic parameters in patients with COVID-19 infection. Am J Hematol. 2020; 95(6):E131-134.

Gattinoni L, Coppola S, Cressoni M, Busana M, Rossi S, Chiumello D. Covid-19 Does Not Lead to a "Typical" Acute Respiratory Distress Syndrome. Am J RespirCrit Care Med. 2020;201(10):1299-1300.

Huang I, Pranata R. Lymphopenia in severe coronavirus disease-2019 (COVID-19): systematic review and meta-analysis. J. Intensive Care. 2020;

Lang M, Som A, Mendoza DP, Flores EJ, Reid N, Carey $\mathrm{D}$, et al., Hypoxaemia related to COVID-19: vascular and perfusion abnormalities on dual-energy CT. Lancet Infect Dis. 2020 (in press).

Litton E, Lim J. Iron metabolism: an emerging 
therapeutic target in critical illness. Crit Care Critical Care. 2019;23:1-8.

Liu R, Wang Y, Li J, Han H, Xia Z, Liu F, et al., Decreased $\mathrm{T}$ cell populations contribute to the increased severity of COVID-19. ClinChimActa. 2020;508:110-4.

Pietrangelo A. Pathogens, metabolic adaptation, and human diseases - an iron-thrifty genetic model. Gastroenterology. 2015;149:834.

Richardson S, Hirsch JS, Narasimhan M, Crawford JM, McGinn T, Davidson KW, et al., Presenting characteristics, comorbidities, and outcomes among 5700 patients hospitalized with COVID-19 in the New York City Area. JAMA. 2020 (in press).

Stites SW, Nelson ME, Wesselius LJ. Transferrin concentrations in serum and lower respiratory tract fluid of mechanically ventilated patients with COPD or ARDS. Chest. 1995;107:1681-5.

Vaira, L.A., G. Salzano, G. Deiana, G. De Riu Anosmia and ageusia: common findings in COVID-19 patients Laryngoscope, 130 (2020) 1787-1787.

Velavan TP, Meyer CG. Mild versus severe COVID-19: laboratory markers. Int J Infect Dis. 2020; 95: 304-7.

Wan S, Yi Q, Fan S, Lv J, Zhang X, Guo L, et al., Relationships among lymphocyte subsets, cytokines, and the pulmonary inflammation index in coronavirus (COVID-19) infected patients. Br J Haematol. 2020; 189: 42837.

Wang F, Nie J, Wang H, Zhao Q, Xiong Y, Deng $\mathrm{L}$, et al., Characteristics of peripheral lymphocyte subset alteration in COVID-19 pneumonia. J Infect Dis. 2020; 221: 1762 9.
Wang L, Duan Y, Zhang W, Liang J, Xu J, Zhang $\mathrm{Y}$, et al., Epidemiologic and clinical characteristics of 26 cases of Covid-19 arising from patient-to-patient transmission in Liaocheng. China ClinEpidemiol. 2020;12:387-391.

Wang L, He W, Yu X, Hu D, Bao M, Liu H, et al., Coronavirus disease 2019 in elderly patients: Characteristics and prognostic factors based on 4-week follow-up. J Infect. 2020;80(6):639-645.

Wang W, Chen S, I-Jun L, Kao C, Chen H, Chiang B, et al., Temporal relationship of viral load, ribavirin, interleukin (IL)-6, IL8 , and clinical progression in patients with severe acute respiratory syndrome. Clin Infect Dis. 2004; 39:1071-5.

Yang J, Zheng Y, Gou X, Pu K, Chen Z, Guo Q, et al., Prevalence of comorbidities and its effects in coronavirus disease 2019 patients: a systematic review and metaanalysis. Int J Infect Dis. 2020; 94:91-95.

Yilmaz, B., Hai Li Gut microbiota and iron: the crucial actors in health and disease Pharmaceuticals, 11 (2018), p. 98

Zhou F, Yu T, Du R, Fan G, Liu Y, Liu Z, et al., Clinical course and risk factors for mortality of adult inpatients with COVID19 in Wuhan, China: a retrospective cohort study. Lancet. 2020; 395 (10229): 10541067.

Zhou, F., T. Yu, R. Du, G. Fan, Y. Liu, Z. Liu, et al., Clinical course and risk factors for mortality of adult inpatients with COVID19 in Wuhan, China: a retrospective cohort study Lancet, 395 (10229) (2020), pp. 1054-1062.

\section{How to cite this article:}

Neha Singh, Dinesh Kumar and Rammilan. 2020. Anemia and Iron Metabolism in COVID-19. Int.J.Curr.Microbiol.App.Sci. 9(09): 3056-3060. doi: https://doi.org/10.20546/ijcmas.2020.909.377 